\title{
INVERSE PROBLEMS FOR A CLASS OF STURM-LIOUVILLE OPERATORS WITH THE MIXED SPECTRAL DATA
}

\author{
YU PING WANG
}

Abstract. In this paper, we study the inverse spectral problem for Sturm-Liouville equations with boundary conditions polynomially dependent on the spectral parameter and establish a uniqueness theorem with the mixed spectral data. In addition, we obtain three corollaries of the uniqueness theorem for the above boundary value problem.

Mathematics subject classification (2010): 34A55, 34B24, 47E05. value.

Keywords and phrases: Inverse problem, Sturm-Liouville operator, spectral parameter, potential, eigen-

\section{REFERENCES}

[1] P. A. Binding, P. J. Browne And K. Seddighi, Sturm-Liouville problems with eigenparameter dependent boundary conditions, Proc. Roy. Soc. Edinburgh, 37 (1993), 57-72.

[2] G. BorG, Eine Umkehrung der Sturm-Liouvilleschen Eigenwertaufgabe, Acta Math., 78(1946), 1-96.

[3] A. Y. Chernozhukova And G. Freiling, A uniqueness theorem for inverse spectral problems depending nonlinearly on the spectral parameter, Inverse Probl. Sci. Eng., 17 (2009), 777-785.

[4] G. FREILING AND V. A. YURKO, Inverse problems for Sturm-Liouville equations with boundary conditions polynomially dependent on the spectral parameter, Inverse Problems, 26 (2010), 055003 (17 pp).

[5] C. T. Fulton, Two-point boundary value problems with eigenvalue parameter contained in the boundary conditions, Proc. Roy. Soc. Edinburgh, 77a (1977), 293-308.

[6] F. Gesztesy And B. Simon, Inverse spectral analysis with partial information on the potential, II. The case of discrete spectrum, Trans. Amer. Math. Soc., 352 (2000), 2765-2787.

[7] H. Hochstadt And B. LieBerman, An inverse Sturm-Liouville problem wity mixed given data, SIAM J. Appl. Math., 34 (1978), 676-680.

[8] M. HoRvath, On the inverse spectral theory of Schrödinger and Dirac operators, Trans. Amer. Math. Soc., 353 (2001), 4155-4171.

[9] V. A. MARChenko, Some questions in the theory of one-dimensional linear differential operators of the second order, I, Trudy Moskov. Mat. Obsc. 1 (1952), 327-420 (Russian); English transl. in Amer. Math. Soc. Trans1., 2, 101 (1973), 1-104.

[10] V. PivovarchiK, On the Hald-Gesztesy-Simon theorem, Integral Equations and Operator Theory, 73 (2012), 383-393.

[11] C. T. Shieh, S. A. Buterin, And M. Ignatiev, On Hochstadt-Liberman theorem for SturmLiouville operators, Far East J. Appl. Math., 52 (2011), 131-146.

[12] T. SUZUKI, Inverse problems for heat equations on compact intervals and on circles, I, J. Math. Soc. Japan., 38 (1986), 39-65.

[13] Y. P. WAng, Uniqueness Theorems for Sturm-Liouville Operators with Boundary Conditions Polynomially Dependent on the Eigenparameter from Spectral Data, Results in Mathethmatics, 63 (2013), 1131-1144.

[14] G. S. WEI AND H. K. XU, Inverse spectral problem with partial information given on the potential and norming constants, Trans. Amer. Math. Soc., 364 (2012), 3265-3288.

[15] C. F. YANG AND X. P. YANG, Inverse nodal problem for the Sturm-Liouville equation with polynomially dependent on the eigenparameter, Inverse Probl. Sci. Eng., 19 (2011), 951-961. 
[16] V. A. YURKo, Method of Spectral Mappings in the Inverse Problem Theory (Inverse and Ill-posed Problems Series), Utrecht: VSP, 2002. 